ce fut tout; les aulres restèrent à l'état de manuscrit, s'échelonnant des années r 862 à r 902 ; ils sont, comme je l'ai dit, conservés au Ministère de l'Agriculture, auquel furent attribués, dès sa création, les cours d'eau non navigables ni flottables. Si les cartes départementales, pour lesquelles une échelle unique n'a pas été adoptée, laissent souvent à désirer, les états $A$ et $B$ permettent d'arriver à une connaissance certaine des meilleures rivières d'un département.

Le premier de ces tableaux (A) est consacré aux données dites permanentes des cours d'eau, classés méthodiquement, comme dans la carte produite, c'est-à-dire que le $\mathbf{n}^{\mathbf{0}} \mathbf{x}$ était attribué au fleuve principal, ou au premier de ses affluents sortant du département pour l'atteindre, et les numéros suiyants épuisaient tous ses affluents jusqu'au dernier avant de passer à un autre cours d'eau tributaire du fleuve $\left.{ }^{1}\right)$.

S'il faut faire quelque réserve en ce qui concerne les débits qui n'ont pu être étudiés avec la précision actuelle, une table alphabétique permel la recherche rapide de toutes les rivières, voir même... des ruisseaux ! Ainsi, le zèle de l'Administration a été bien loin, ct l'on peut être étonné de trouver dans ces tables, par exemple, l'égoul des fortifications de certaine ville du Nord, ou les 2,26 cours d'eau... anonymes du département de l'Héraull qui, cependant, ont tous leurs situations bien définies, grâce à la méthode sus-mentionnée. Par contre, si l'état $\mathbf{B}$, dit des Usines et Irrigations, pèche parfois par une date un peu reculée, on peut avoir foi en lui, pour évaluér la qualité et la constance de la rivière, si j'ose dire. En effet, pour fixer la puissance d'une usine hydraulique, on pouvait avoir recours à l'estimalion du moteur établi, estimation qui avait pu êlre confiée à un employé subalterne. Puisque, pour sélectionner mes cours d'eau, j'avais adopté un critérium, assez peu élevé, 25 chevaux, c'est donc sur cette basse la plus ceriaine de la force utilisée. que j'arêtais mon choix. La suite justifiait ce critérium puisque, grâce encore au concours administratif, je relevais des distributions publiques d'énergie élcctrique, sur presque toutes ces rivières.

Voici comment l'Administration a bien voulu me faciliter la recherche des distributions publiques hydro-électriques. Une première fois, à l'occasion de l'Exposition internationale de Bruxelles rgog, la Direction de l'Hydraulique Agricole prit la peine, en présence de mes efforts, et dans le but d'en exposer une carte murale, de demander aux ingénieurs en chef de chaque département du bassin de la Loire de corriger s'il y avait lieu, ct surtout de compléter le premier résultat de mes recherches. L'année rgro, ce fut le tour du Bassin de la Garonne, pour l'Exposition de Turin. L'enquếte générale se trouvait ainsi amorcée en quelque sorte, et les bassins de la Seine et du Rhône avaient subi la même revision ; actuellement, la dernière partie, celle des bassins côtiers ou frontières, est soumise au même examen, et la Dircction Générale des Eaux et Forêts disposera, pour une exposition future, d'un ensemble complet, inédit et garanti pour toule la France.

Rappelons sommairement les bases de la carte produite : $I^{\circ}$ Toute rivière ayant eu une utilisation hydraulique de 25 chevaux au moins, avec toutes les distributions publiques hydro-électriques, qu'elles disposent ou non d'un secours à vapeur plus ou moins important; $2^{\circ}$ Egalement, toute rivière qui, n'étant pas dans cette première condition, fournit

(1) Sur la carte cimjointe, le cours d'eau numéroté 9 représente un canal dérivé du Verdon, et amenant une partie des eaux du Verdon hors du bassin du Rhône. actuellement une énergie hydraulique d'au moins 25 chevaux à une distribution publique ; elles sont au nombre de 74 et, avec les premières, forment un total de rgr. Je terminerai en faisant remarquer qu'il y a encore, dans le bassin du Rhône; 44 cours d'eau, alimentant 49 de ces usines, qui ont moins de 25 chevaux et qui, par conséquent, ne figurent pas sur la carte.

Henri Bresson.

\section{COMPTEURS VENTURI GIGANTESQUES DU SERVICE DES EAUX DE NEW-YORK}

Par suile de laccroissement constanl de la population de New-York, le Service des Eaux de cetle ville a dû entreprendre de nouveaux travaux pour pouvoir amener à bref délai de nouvelles quanlités d'eau potable, malgré les réserves déjà considérables accumulées dans les nombreux réservoir's qui l'alimentent ${ }^{1}$ ). Pour cela, le Service des Eaux de NewYork fait actuellement construire le grand barrage-réservoir d'Ashokan (voir La Ilouille Blanche de novembre rgo7), ainsi que l'aqueduc de Catskill, qui doil amener à New-York les eaux de ce nouveau réservoir.

Cet aqueduc est remarquable par les nombreux travaux d'art qu'a nécessité sa construclion, el par les divers ouvrages accessoires qui l'accompagnent. La Houille Blanche de janvier ı 9 r 2 a signalé notamment ses siphons métalliques sous pressions, avec revêtements cimentés, tant à l'intérieur qu'à l'cxtérieur, ainsi que les vannes de réglage du débit, à la sortie du réservoir d'Ashokan. Nous nous proposons aujourd'hui de décrire, d'après l'Engineering Record du 20 janvier $19 \mathrm{r}_{2}$, l'installation de trois gigantesques compteurs d'eau, du système Venturi, qui ont été installés en trois points différents de cet aqueduc, el qui sont bien, vraisemblablement, les plus grands compteurs de ce système, qui aient jamais été construits dans le monde cntier $\left(^{2}\right)$.

L'aqueduc de Catskill part du réservoir d'Ashokan, qui est à r 30 kilomètres au nord de New-York, el amène l'eau par la seule gravité au réservoir de Kensico, qui n'est qu'à 50 " km. de New-York. De ce réscrvoir de Kensico, l'eau est amenée au réservoir répartiteur de Hill View, pour être distribuée ensuite par toute la ville. Cet aqueduc a été prévu pour pouvoir débilcr un volume journalier de 500 millions de gallons, ce qui correspond à r 893 ooo mètres cubes par jour ou à 22 mètres cubes par seconde.

Un premier compteur Venturi est installé au départ de l'aqueduc dès qu'il vient de recevoir les eaux du réservoir d'Ashokan ; le second et le troisième compteur sont placés respectivement à l'entrée et à la sortic du réservoir de Kensico. De cette manière, le compleur placé à la sortie du. réservoir de Kensico permeltra de délerminer quel est le volume d'eau fourni à la ville de New-York par le dit réser-

(1) A l'heure actuclle, la ville de New*York est principalement alimentée par l'aqueduc du Croton, qui peut débiter jusqu'à 330 millions de gallons par jour. Cet aqueduc part du grand réservoir du Croton, qui, avec 15 autres réservoirs répartis à travers le bassin hydrologique du Croton, utilisent à peu près intégralement la totalité de l'eau débitée annuellement par cette rivière.

Le dernier recensement de l'agglomération de New-York (1910) indique une population de 6.475 .000 habitants. En r 1900 , il n'y avait que 4.608 .000 habitants. L'augmention a doncété de 40 pour 100 pendant ces dix dernic̀res années.

(2) On trouvera dans La Houille Blanche de mai 1909 une étude sur le compteur Venturi, ainsi que le compte rendu d'essais faits, sur ce système de compteurs, au Laboratoire d'essais du Conservatoire national des Arts et Métiers, par M. Boyer-Guillon. 
voir. Lc compteur placé en amont de ce réservoir, combiné avec celui placé en tête de l'aqueduc, permettra au Service des Eaux, non seulement de connaître le volume d'eau débité par l'aqueduc dans un temps donné, mais encore de se rendre compte de l'importance des fuites, défalcation faite des infiltrations qui pourraient se produire.

Les trois compteurs Venturi sont identiques, et sont en béton armé. Afin que ces compteurs soient certainement toujours pleins d'eau, on les a intercalés chacun dans un siphon renversé, de 408 pieds $\mathrm{r} / 2$ de longueur, soit I $25 \mathrm{~m}$., et disposé de telle sorte qu'il y ait toujours, au-dessus de la partie centrale du compteur, une pression de 18 pieds $\left(0^{\mathrm{m}} 55\right)$. trale, et 16 pour le cône d'amont. Ces trous ont été percés bien perpendiculairement à la surface intérieure, qui a été ensuite soigneusement polie. En face de chaque trou, et sur la paroie extérieure des chambres de pression, on a disposé un tube (pressure pipe connection), fermé normalement par un chapeau, et sur lequel on peut brancher éventuellement un manomètre.

L'appareil de mesure proprement dit est composé essentiellement d'un tube en $U$, contenant du mercure, et dont l'une des branches est reliée avec la chambre de pression annulaire amont, tandis que l'autre branche est reliée à la chambre de pression centralc. Ainsi qu'on le sait, la diffé-

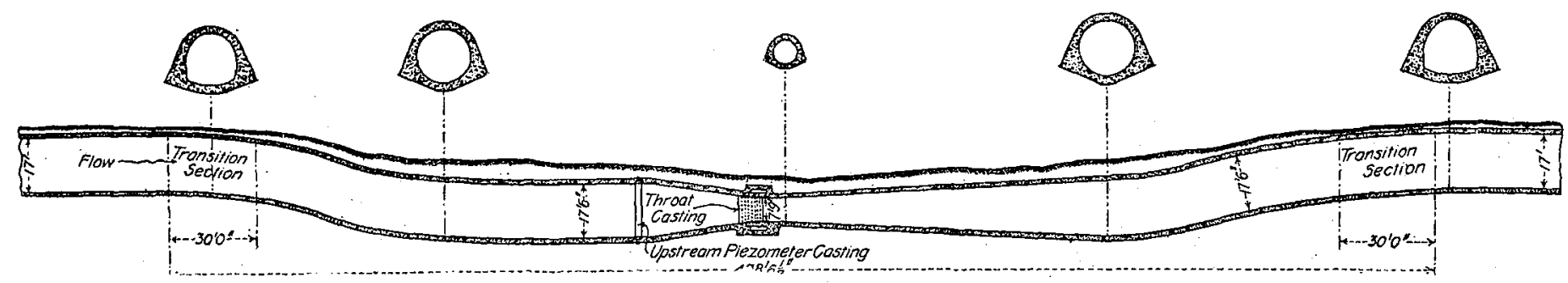

FIG. I. - Coupe en long montrant la position du compleur Venturi dans le siphon renversé.

Pour chaque compteur, le còne d'amont a une longueur de 26 pieds 3 pouces $5 / 8\left(8^{\mathrm{m}} \mathrm{O} / 7\right)$, et celui d'aval, une longueur de in I pieds 7 pouces $7 / 8\left(34^{\mathrm{m}} \mathrm{o} 32\right)$. Le diamètre intérieur de la partie centrale est de 7 pieds 9 pouces, soit $2^{\mathrm{m}} 362$, et le plus grand diamètre des cônes amont et aval, qui est on même ternps celui du siphon, est de 17 pieds 6 pouces, soit $5^{\mathrm{m}} 334$. rence des vilesses de l'eau devant chacune des chambres de pression produit une certaine dénivellation du mercure dans les deux branches du tube en $\mathrm{U}$, et toute variation dans le débit de l'aqueduc se traduit par une variation de cette dénivellation. Le mouvement du mercure, dans chaque branche, entraîne celui d'un flotteur, et, au moyen d'un dispositif approprié, ces flotteurs agissent à leur tour sur les aiguilles
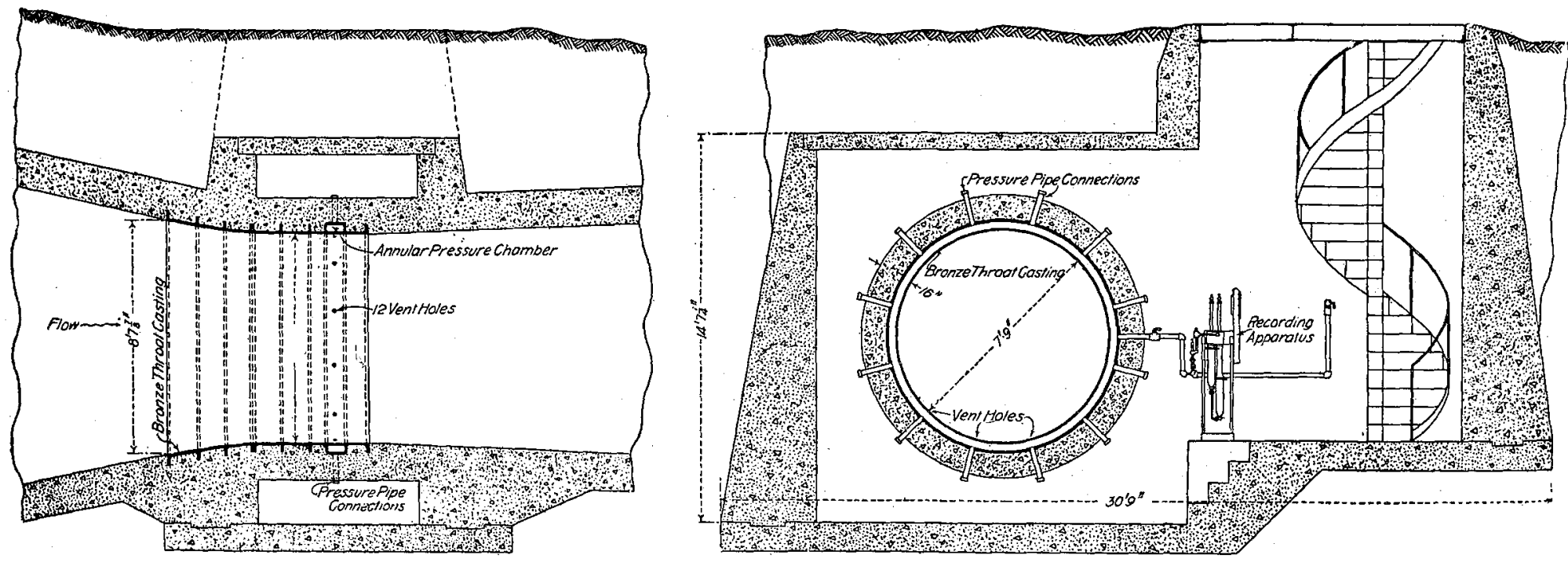

FIG. 2. - Coupes en long el en travers de la partie centrale da Compteur Venluri.

Afin d'avoir une surface inléricure toujours nette, et un diamètre parfaitement déterminé, on a muni l'entréc du còne amont, ainsi que la partie cenlrale el son raccordement avec le còne amont, d'une garniture en bronze, qui porte, venue de fonte avec elle, une chambre de pression annulaire. La garniture d'amont (upstream piezometer casting) est disposée à 5 pieds ( $\left.{ }^{\mathrm{m}} 525\right)$ de l'extrémité amont du cône convergent. Elle a une largeur de 12 pouces $\left(\mathrm{I}^{\mathrm{m}} 305\right)$ et est constituée par 16 segments boulonnés à la suite les uns des autres. La gamiture centrale (bronze throal casting), qui est représentée en coupe longitudinale sur la figure 2, a une longueur de 7 pieds 6 pouces $\left(2^{\mathrm{m}} 286\right)$.

Les deux chambies te pression annulaires sont en communication avec lo courant d'eau au moyen d'un certain nombre de trous (vent holes), savoir : r 2 pour la partie cen- de deux cadrans. Liun de ces cadrans indique simplement le débit de l'eau à un instant donné ; l'aulre est enregistreur' (recording apparatus) et inscrit le mouvement des eaux sur' une feuille de papier pour une semaine entière.

Cet appareil de mesure est installé dans une petite chambre en béton, qui est disposée immédiatement à côté de la partie centrale du tube Venturi, ainsi qu'on peut le voir sur la figure 2.

Ces compteurs Venturi ont été étudiés par le bureau technique du Service des Eaux de New-York, en collaboration avec la Builders Iron Foundry, de Providence (Rhodes Island), qui a fondu les garnitures des chambres de pression, et qui est l'un des principaux constructeurs de compteurs Venturi en Amérique.

H. B. 